\title{
La arqueología patagónica y la reconstrucción de la historia indígena
}

\author{
$\mathrm{M}^{\mathrm{a}}$ Florencia del CAstillo Bernal \\ florenciadelcastillo@hotmail.com \\ Laura MAMELI \\ Universidad Autónoma de Barcelona \\ laura.mameli@uab.cat \\ Joan A. BARCELÓ \\ Universidad Autónoma de Barcelona \\ juanantonio.barcelo@uab.cat
}

Agencia Española de Cooperación Internacional y Desarrollo - Universidad Autónoma de Barcelona

Recibido: 2 de diciembre de 2009

Aceptado: 9 de octubre de 2010

\section{RESUMEN}

En este trabajo realizamos una revisión de las explicaciones tradicionalmente brindadas por la arqueología y la antropología patagónicas a los procesos de construcción de la diversidad y la homogeneización social. Al margen del concepto de «Cultura Arqueológica», intentamos buscar alternativas basadas en una concepción distinta de la «etnicidad» en sociedades cazadoras-recolectoras, tomando en consideración la dinámica característica de los diferentes procesos de interacción posibles, así como los mecanismos de agregación/segregación y la conformación irregular y cambiante de normas sociales. Para ello se propone como metodología más adecuada la simulación computacional distribuida, o sociedad artificial.

Palabras clave: Patagonia, arqueología, cazadores-recolectores, historia indígena, etnicidad, etnogénesis.

\section{Patagonian Archaeology and the Reconstruction of Indigenous History}

\begin{abstract}
In this paper we review archaeological and anthropological traditional explanations of diversity formation and social homogeneity in Patagonia. In spite of «Archaeological Culture» concept, our goal is to look for alternatives based upon a different concept of what «ethnicity» is among hunter-gatherer societies. This new perspective considers the characteristic dynamics of the different possible social interaction processes, the aggregation/segregation mechanisms and the irregular and changing formation process of social norms. As methodology, we suggest distributed computational simulation that is the building of artificial societies.
\end{abstract}

Key words: Patagonia, archaeology, hunter-gatherers, indigenous history, ethnicity, ethnogenesis.

SUMARIO: 1. Introducción. 2. Las primeras etnografías. 3. Las primeras investigaciones arqueológicas. 4. Teorías histórico-culturales. 5. Menghin y la Arqueología Histórico-Cultural. 6. La reacción en contra de la explicación histórico-cultural. Etnohistoria e historia indígena. 7. La arqueología procesual y el neoevolucionismo en la arqueología patagónica. 8. ¿Es posible una interpretación alternativa en la arqueología de Patagonia? 9. Conclusiones. 10. Referencias bibliográficas.

\section{Introducción}

En los comienzos de la etnología patagónica (siglos XIX-XX), el desarrollo de las investigaciones acompañó el proceso de conformación de una historiografía nacional argentina que, en términos de Eric Wolf (1982), presentaba a las sociedades colonizadas como meros apéndices de las historias nacionales. La mayoría de los estudios 
históricos para Patagonia concentraban sus intereses en la guerra de frontera, principalmente de los siglos XVIII y XIX, en los que se identificaba a los aucas ${ }^{1} \mathrm{o}$ araucanos como los indios de presencia chilena en una Pampa-Patagonia considerada necesariamente argentina.

El modelo europeo de estado moderno sustentado por una burguesía terrateniente, defendía sus intereses políticos y económicos justificando el avance de frontera y el etnocidio indígena. La institucionalización de un corpus político-legal permitió la fácil obtención de mano de obra, proletarizando a los pobladores originarios junto con ciertas remesas emigrantes (Gómez Romero 2007). Este mismo modelo legitimó la idea de un territorio desierto, avalando de este modo la apropiación y obtención de tierras, desintegrando las estructuras sociales de sus pobladores originales al transformarlos en proletariado agrario (Bartolomé 2004; Gómez Romero 2007; Mandrini 1988). Este brusco y forzado proceso de incorporación al mercado laboral como mano de obra asalariada mal pagada, junto con un discurso nacional que sostenía una idea de homogenización nacional basada en la idea de una Europa blanca y civilizada, condujeron a la desvinculación del indígena de la historia nacional. Aun cuando los respectivos ejércitos nacionales integraban en sus filas a gauchos, criollos, españoles o indios amigos, dentro del contexto de construcción ideológica estatal, el indio era el reflejo del vago, ladino, ladrón, sanguinario, adjetivos que se harán extensibles a todo el inmenso y multiforme proletariado de frontera. Paralelamente, mientras la historiografía argentina construye la historia nacional, vincula inmediatamente a los indígenas más reticentes al proceso de expansión de la frontera sur como chilenos, enlazando la cuestión indígena con la oposición argentino-chilena, fenómeno característico del proceso de formación de las identidades nacionales durante el siglo XIX.

Esta progresiva invisibilización del indígena por parte del Estado, legitimó el proceso de extinción de los pueblos indígenas. Al convertirse en un país en «blanco», el estudio de Patagonia fue relegado al anticuarismo y a la mera curiosidad por el conocimiento de sociedades que se consideraban ya desaparecidas. En sus comienzos la etnología y la arqueología profesionales acompañaron esta conformación de los discursos nacionales, incorporando el estudio de los pueblos indígenas al ámbito de los museos y academias científicas, naturalizando la imagen del indígena como un componente más a registrar del mundo natural. Detrás de este distanciamiento, diferenciación y delimitación del objeto de estudio, subyace una argumentación que naturaliza una inferioridad biológica, consecuencia de un pensamiento racista que no siempre es explicitado por parte de la comunidad académica (Curtoni y Politis 2006).

A pesar de la fuerte crítica que se ha realizado de esta postura teórica, la concepción de unidades cerradas, inmutables e ideales de las trayectorias históricas y la conformación social indígena aún pervive. El peso de los discursos descriptivistas, tipologicistas y evolucionistas replican una continuidad histórica de poblaciones cazadoras recolectoras a lo largo de 13.000 años de historia. La crítica y consecuente sustitución de la categoría «cultura arqueológica» por «etnia», «sistema cultural»o «modo de

\footnotetext{
${ }^{1}$ Auca: enemigo-rebelde en quechua. Denominación dada por los Incas a los pueblos que habitaban el sur del río Maipo (Chile), continuó siendo utilizada por los españoles durante los siglos XVI y XVII de manera generalizada para denominar a los indígenas trasandinos.
} 
producción» no ha conducido a una renovación de los principios subyacentes; la permanencia del estigma de la identidad cultural degradada, el sometimiento político y la marginación aún perviven.

\section{Las primeras etnografías}

En las primeras décadas del siglo XX comienzan los estudios etnográficos a partir de los trabajos de Milcíades A. Vignati (1936), Tomás Harrington (1946) y Federico Escalada (1949), quienes, sirviéndose de fuentes escritas y de su propio trabajo de campo, intentaron unificar, diferenciar y clarificar el panorama indígena; sus trabajos apuntan a clasificaciones fundamentadas en la asociación de los conceptos de raza, cultura y territorio. Vignati, por ejemplo, proponía que todo elemento considerado culturalmente complejo era resultado de las relaciones con pueblos del oeste cordillerano, creyendo que las innovaciones dadas en las denominadas altas culturas se habrían difundido hacia el sur-sureste, actuando como estímulo externo (Vignati 1944). En la mayoría de sus investigaciones se sirve de fuentes históricas y de segunda mano, centrando principalmente su interés en la recopilación de datos empíricos, la elaboración de detalladas descripciones y el afán por realizar síntesis interpretativas que le permitieran establecer los límites de las etnias conocidas históricamente para Patagonia: pampas, tehuelches y araucanos (Nacuzzi 2005).

Entre los primeros registros etnográficos propiamente dichos figuran los de Federico Escalada ${ }^{2}$, quien durante los años que vivió en la localidad de Río Senguer, Chubut, elaboraría su esquema etnológico y trabajaría en la recopilación de un registro oral. Este autor propone una división étnica entre grupos mapuche o araucano, complejo tehuelche y fueguino, fundamentada en diferencias referentes a rasgos lingüísticos, culturales y raciales. Para Escalada, la denominación de complejo tehuelche contempla la inclusión de grupos günuna'kénk o tehuelche septentrional, aónik'kénk o tehuelche meridional, chehuache'kénk, selk'nam y man'kénk ${ }^{3}$. En su publicación sobre las cuencas hidrográficas patagónicas (Escalada 1949) enfatiza la vinculación entre etnia y territorio, definiendo áreas de circulación de los grupos tehuelche a través de las principales cuencas hidrográficas patagónicas.

En los trabajos de Tomás Harrington encontramos un exhaustivo registro de la lengua günün a iajüch del grupo autodenominado günuna 'kénk de Patagonia septentrional. Este autor, siendo inspector de escuelas en la meseta central de Chubut, no solo realiza un registro de la lengua que recoge a lo largo de los quince años de trabajo en Patagonia (1911-1936), sino que como parte de su obra recoge leyendas, canciones y

\footnotetext{
${ }^{2}$ Médico de la Gendarmería Argentina en la Gobernación Militar de Comodoro Rivadavia.

${ }^{3}$ Los primeros etnógrafos realizaron transcripciones fonéticas en las que el sonido indígena se hacía corresponder a la fuerza al sonido de la lengua castellana (o inglesa) que más se parecía a juicio subjetivo del que lo transcribía, por lo cual tanto etnónimos y glotónimos se presentan en la bibliografía de diversas maneras. Aunque la lingüística patagónica es hoy una disciplina bien establecida, falta una normalización fonética (véase Viegas Barros 2005). En este artículo hemos tomado la opción de tipificar etnónimos y glotónimos de acuerdo con las reglas más usuales en la etnografía actual, aunque somos bien conscientes que hemos optado por la solución más simple, y no la más correcta (cf. Barceló, del Castillo, Mameli, Moreno y Videla 2009).
} 
referencias toponímicas, con referencias en lenguas mapuche, günün a iajüch y español. Tanto Harrington como Escalada, a pesar de no ser etnógrafos profesionales y de estar ellos mismos inmersos dentro de los postulados anticuaristas y tipologicistas, intentaron poner de manifiesto aquello que aún sobrevivía de las sociedades indígenas, convirtiéndose sus etnografías en las mejores descripciones disponibles sobre los cambios sufridos después de la conquista.

Estas primeras etnografías tuvieron lugar ya avanzada la desarticulación de la sociedad indígena y la justificación del orden estatal. Para entonces los indígenas representaban un objeto-rareza en vías de extinción que demandaban un rescate de índole prioritariamente simbólico y cultural. La necesidad de rescatar al último tehuelche, conduce a una afirmación implícita sobre la inmutabilidad de la población indígena, y al mismo tiempo hacia una exclusión dentro del proyecto nacional en construcción.

A modo de síntesis podemos decir que estos tres autores coinciden en plantear una definición sobre la organización social tehuelche basada en rasgos raciales, culturales y lingüísticos, y cuya ordenación geográfica permitió la delimitación de las fronteras entre las diferentes parcialidades étnicas. Los límites interétnicos reflejaban entonces las coincidencias con los mosaicos ambientales, concibiendo de este modo al territorio de manera equivalente tanto con el ambiente y con los grupos étnicos. En el análisis de la sociedad indígena, la idea de simplicidad continuó la propuesta de las fuentes históricas, y la herencia del paradigma darwiniano se expandió a todas las etnias del territorio. Más allá de que el mismo Darwin observara y describiera sociedades bien diferenciadas, la concepción evolutiva de simplicidad descrita para los canoeros del extremo austral, sumado a las extremas limitaciones ambientales o «territorio maldito» -palabras de Darwin-, se trasladaron a la descripción de todo el ámbito patagónico reproduciéndose así en la bibliografía etnográfica.

Señalamos separadamente los trabajos de Rodolfo Casamiquela por entenderlos como una pervivencia de esta manera de hacer etnografía y servir de eje articulador entre las primeras prácticas etnográficas y la Escuela Histórico Cultural. Formado como autodidacta, sus investigaciones comienzan con un análisis dentro del campo de la paleontología, volcándose luego hacia la etnografía y la lingüística. El pensamiento que subyace dentro de toda su obra es el tipológico clasificatorio del anticuarismo y de la Historia Natural de la época de la Ilustración. Traslada los formatos de clasificación linneanos que utiliza como paleontólogo a la conformación de tipologías étnicas que vinculan lengua, cultura, raza y territorio. En su esquema de clasificación propuesto para el siglo XVIII-XIX (Casamiquela 1965) utiliza fuentes históricas de las que extrae información lingüística y gentilicia que suma a su propia información etnográfica. Identifica grupos primigenios a partir de su lengua y visualiza la dinámica limitándose a consignar posibles relocalizaciones espaciales, a la vez que cree identificar influencias de etnias «extranjeras», hablantes de otras lenguas. Ejemplo de estas influencias externas sería el caso del pueblo manzanero, que habría incorporado la lengua mapudungün y se situaría en el sur de Neuquén, es decir, una cultura que se habría transformado a partir de las particularidades de su emplazamiento y en lo fundamental por el contacto con otras etnias (Casamiquela 1985).

Claramente, para Casamiquela la variabilidad étnica y territorial del poblamiento autóctono de Patagonia es concebida como un mecanismo sobre la base de conjuntos 
cerrados, cambiantes sólo por causa de la contaminación de las bases primarias de su configuración interna: lengua-raza-territorio-cultura. En sus trabajos, los postulados etnológicos continúan la búsqueda de la tradición nacional, asociando lo tehuelche con la formación de lo patagónico-argentino, y asociando lo mapuche a todos aquellos rasgos considerados chilenos. Tal como dice en sus textos:

«El área más conservativa del norte de la Patagonia fue, desde luego, el corazón del Río Negro y del Chubut. Las influencias de los indígenas sur-neuquinos, sensu lato, y Araucanos veros, que llegaban por el noroeste y oeste, no trascendían seguramente de Maquinchau hacia el interior, por lo menos de manera significativa. Mayor contaminación había -curiosamente- con los Tehuelches meridionales» (Casamiquela 1985:4).

La constante búsqueda de orígenes de poblaciones puras le impide concebir la transformación histórica del indígena y aceptar el mestizaje. La posibilidad de encontrar algún elemento de diferenciación social implicaba haber encontrado un rasgo definitorio del cambio, entendido este como reemplazo poblacional y vinculándolo a diferencias nacionales: tehuelches/argentinos - mapuches/chilenos. A pesar de la lectura lineal de sus planteamientos y de las fuertes críticas pronunciadas sobre la empiria histórica y etnográfica, y sobre la realización de asociaciones lingüísticas directas (Nacuzzi 1998; Vezub 2005), o sobre la desactualización de los argumentos antropológicos que refutan las asociaciones entre raza-cultura (Cocilovo 1992), desde el campo arqueológico su esquema es entendido como el resultado final del devenir histórico de las sociedades indígenas y como el reflejo de las relaciones vigentes en el proceso final de la desarticulación de las poblaciones originarias.

\section{Las primeras investigaciones arqueológicas}

Los primeros naturalistas del siglo XIX, en su afán por reunir información para los museos, desenterraron restos de objetos y esqueletos de los que consideraban exponentes de «razas en vías de extinción», historias ajenas, pasadas y superadas. A fines del siglo XIX y principios del XX, la arqueología tampoco fue ajena al lugar que le cupo a las ciencias sociales dentro del proyecto liberal de nación: proporcionar evidencias sobre el triunfo del Orden sobre el salvaje y de la Cultura sobre la ignorancia, legitimar políticamente un sistema de Estado moderno y establecer las bases de la identidad nacional. Los trabajos de estos naturalistas contribuyeron a la progresiva institucionalización y al reconocimiento de la arqueología para la definición de lo nacional. Trabajos como los de Francisco Ambrosetti (1903), Francisco de Aparicio (1935), Carlos Bruch (1902), Félix Outes (1905), Milcíades Vignati (1936), intentaban definir las etnias a partir de su emplazamiento geográfico y a las sociedades consideradas prehistóricas en cuanto a las asociaciones geocronológicas. Criticaban las interpretaciones directas de las fuentes históricas, abogando por la búsqueda de evidencia arqueológica proveniente del registro de colecciones privadas y públicas o de recolecciones de material superficial.

En La edad de piedra en la Patagonia, Outes (1905) recopila y sintetiza la información arqueológica conocida hasta ese momento. Basándose en la periodización del 
Viejo Mundo, define un período Neolítico Patagónico y distingue entre Protoneolítico, Miolítico y Epineolítico, sobre la base de los caracteres tipológicos de los instrumentos de piedra. El eje de análisis es la evolución tecnológica, considerando los cambios, aparición y variación del instrumental como evidencia de desarrollo social. De este modo, explicaciones como la aparición de la industria neolítica de piedra pulida transcordillerana habrían dado lugar a la etapa neolítica de los momentos de contacto, hecho que habría de ser demostrado por la presencia de bolas, manijas, morteros, manos de mortero. A partir de aquí, publicaciones como Los aborígenes argentinos, una sintesis del estado actual del conocimiento de los pueblos indígenas, de Outes y Bruch, cristalizan la idea según la cual las innovaciones dadas en las denominadas altas culturas se habrían difundido hacia el sur-sureste, actuando como estímulo externo para el desarrollo, idea que perdura hasta bien entrado el siglo XX.

En la medida en que estos trabajos configuraron la transición hacia una arqueología moderna, los límites disciplinares fueron cimentando una disciplina que aún hoy sigue basándose en la imagen del extraño, de los otros. La recolección de piezas arqueológicas sigue siendo el testigo de lo pasado, de lo antiguo, de lo que no está, y la práctica arqueológica confirma el vaciamiento de una región y ratifica la ocupación del Estado sobre un verdadero desierto. Esta forma de hacer arqueología entiende a los que no están, los que desparecieron, como un fenómeno propio de la historia patagónica; los indígenas visualizados serían entonces los llegados en momentos posteriores a la definición de las naciones, planteando una ruptura entre prehistoria y presente etnológico.

\section{Teorías histórico-culturales}

La Escuela Histórico Cultural o Escuela de Viena, comienza su desarrollo en Argentina desde la llegada al país de los antropólogos italianos José Imbelloni y Marcelo Bórmida y del suizo Oswald Menghin, incorporándose al ámbito académico de la Universidad de Buenos Aires ${ }^{4}$. Los miembros de esta escuela, desde una postura teórica que el mismo Bórmida denominaba Fenomenología Etnológica, basaban su construcción del objeto de estudio en el concepto de mentalidad primitiva definido por LevyBrühl, oponiendo una mentalidad mítica irracional a otra mentalidad racional que se consideraba patrimonio del mundo occidental (Bórmida 1976). De este modo, se centraron en el registro de mitos indígenas por considerarlos la expresión de estructuras mentales esenciales e inmutables en el tiempo. Desde esta perspectiva, la antropología que fuera desarrollada por Imbelloni (1949) y Bórmida debe ser entendida como un trabajo en conjunto, pues mientras que el antropólogo físico registraba la antropometría y las características somatológicas de los grupos indígenas, Bórmida indagaba y registraba mediante entrevistas los rasgos intelectuales que creía esenciales a cada «raza». Ambos entendían que la cultura debía ser analizada tanto desde los compo-

\footnotetext{
${ }^{4}$ Terminada la II Guerra Mundial, el gobierno peronista respaldó la llegada de investigadores europeos al país ofreciéndoles cargos en las universidades, como el caso de Vladimiro Males (yugoslavo), quien dirigiría el Instituto de Etnología de la Universidad de Tucumán, o el del húngaro Miguel De Ferdinand, quien dirigiría el Instituto de Arqueología y Etnología de la Universidad de Cuyo. El mismo Imbelloni sería quien en el año 1947 crearía el Instituto de Antropología de la Facultad de Filosofía y Letras de la Universidad de Buenos Aires.
} 
nentes mentales y culturales, como desde los raciales. Definían culturalmente las regiones a partir de las proyecciones históricas de los grupos conocidos etnográficamente, mediante la utilización de las variaciones lingüísticas descritas por los primeros cronistas que llegaron a la región, valiéndose de sus propios estudios etnográficos realizados en largas temporadas de campo. Explican los procesos de difusión, migración, guerra o invasión como mecanismos causantes del cambio social, cambios que por otro lado son descritos y entendidos como «relictos de sociedades en extinción» que estarían atravesando un período de decadencia que ineludiblemente ocurre en todos los ciclos culturales.

Desde esta corriente de pensamiento también se prestó interés a la influencia que tendría el medio ambiente en el desarrollo de la cultura, considerando la existencia de un principio de cohesión interna determinante de la diferenciación entre pueblos naturales y civilizados. Su interpretación afirmaba que los pueblos patagónicos carecían de este principio, situación que sumada a las consideradas restricciones ambientales devenía en sociedades con una completa falta de progreso (Boschín y Llamazares 1986). Sostenían que los pueblos agrícolas llegados desde la Araucanía a la Patagonia a partir del siglo XVIII pertenecían a un sustrato cultural superior que habría reemplazado gradualmente a los grupos cazadores de la estepa pero cuyo nivel económico, por causa de las restricciones ambientales, se habría adaptado al modo de vida ecuestre y a la crianza de ganado. Este reemplazo o sustitución de una población por otra representaría un proceso gradual, identificado en función al grado de reemplazo de la lengua, difusión de elementos culturales y modificaciones raciales. Curiosamente, a nivel de la organización social, consideraban que la adaptación a un modo de vida ecuestre significaba la pérdida de la estratificación social, rasgo característico del pueblo mapuche o araucano, manteniendo los rasgos más típicos como la platería, los tejidos y sus rituales, pero reemplazando una subsistencia agrícola por una cazadora. Esta contradicción, analizada por Mandrini (1992) y Ortelli (1996), deja en evidencia la profunda influencia de los elementos ideológicos que sustentan sus teorías. La evidencia que ellos mismos generaban estaba demostrando profundos cambios a nivel social, pero la reducción del análisis a identidades esenciales y cerradas sólo les condujo a interpretar la imposibilidad de que sociedades primitivas pudieran mezclarse con sociedades superiores, pues el desarrollo de capacidades mentales, la expresión de sus atributos físicos y el nivel de desarrollo cultural eran consideradas pruebas categóricas de sus teorías. Dentro de este marco las consecuencias de este modo de hacer etnografía han sido muy claras: la institucionalización de una disciplina dedicada a sociedades exóticas, intrínsecamente distintas a la «nuestra», cuyo pasado se supone idéntico a su presente de pobreza inducida, marginación y exclusión social. Así, la etnografía se habría comportado de modo funcional a la desconexión entre el presente etnográfico y el pasado indígena reforzando el paradigma de invisibilización y exclusión de estos pueblos con su propia historia.

\section{Menghin y la Arqueología Histórico-Cultural}

Desde una arqueología histórico-cultural, Oswald Menghin intentaba documentar a través de los restos arqueológicos los ciclos culturales con sus respectivas áreas y 
niveles de desarrollo cultural, reconstruyendo el pasado en términos de presencia o ausencia de determinados rasgos. Presuponía que el resultado de esta distribución se correlacionaba con las entidades pasadas; de este modo el área cultural estaría definida por el conjunto de culturas afines. Si la etnia representaba una cultura expresada en los rasgos materiales de un yacimiento, entonces un conjunto de yacimientos que presentara similitudes podía definir el área de distribución de esa cultura, a la vez que una relación regional entre culturas, como lo ejemplifican las culturas patagónicas Toldense, Jacobacciense o Sanmatiense, entre otras, definidas principalmente sobre la base de la tecnología lítica (Menghin 1952a, 1952b).

Metodológicamente, el trabajo de Menghin se basaba en recolecciones superficiales y sondeos que le permitían reconstruir áreas geográficas en las que se asociaban rasgos tipológicos y geológicos. Entre 1951 y 1952 Menghin amplía sus exploraciones en Patagonia norte desde Viedma hasta Bahía Solano y hacia el interior meridional de la provincia de Santa Cruz. A partir de este viaje plantea una secuencia cultural para Patagonia basada en la definición de siete estilos de arte rupestre (1952a, 1952b, 1957). De los siete estilos propuestos 5 infiere paralelismos con el arte Paleolítico Superior europeo, por ejemplo el motivo negativo de manos o el geométrico simple cuya analogía la encuentra en la cueva de Niaux, en Francia. Menghin consideraba que América había sido poblada por cazadores superiores que habían culminado la etapa de caza y pesca pero que no habían alcanzado la domesticación animal ni la agricultura, considerando a los tehueche del momento de contacto como poseedores de una cultura miolítica fuertemente neolitizada, con adopción tardía de cerámica y fauna doméstica. Por otro lado, las explicaciones basadas en la relación raza-cultura-lengua-territorio son el eje argumentativo de la perspectiva histórico-cultural, como lo expresara su colega Bórmida:

«persistencia de los tipos craneanos pámpidos originarios en el sud; influencia racial de los ándidos (Araucanos) en la Patagonia media y del norte, que origina un tipo craneano pámpido atenuado y andinizado y una gama de mestizos en los cuales la sangre pámpida o ándida predomina en grados distintos (...) Esta sustitución étnica puede fecharse entre los siglos XVI y XVIII» (Bórmida 1953-54).

Así pues, el proceso de araucanización provendría del oeste cordillerano, entendido como las influencias de los agricultores sobre los cazadores recolectores, resultando de este modo «lo tehuelche» producto de la hibridación cultural comenzada a partir del siglo XVII.

Partiendo de premisas metodológicas afines, Carlos Gradín (1985) propone una secuencia temporal algo más desarrollada a partir del arte rupestre y de la tecnología lítica, con el fin de conferir temporalidad al esquema histórico-cultural de la región patagónica. Para el período de contacto señala que la sociedad patagónica o tehuelche estaba compuesta por bandas de cazadores recolectores nómadas que mantenían contactos con sociedades agro-alfareras de la región pampeana y andina. Asume como esencialmente correcta la tipología étnica definida por Casamiquela (1969), diferenciando entre tehuelches septentrionales australes y meridionales -características pro-

${ }^{5}$ Negativos de manos, escenas, pisadas, paralelas, grecas, miniaturas y símbolos complicados. 
pias de los siglos XVI, XVII y XVIII-y la aplica en la diferenciación de las diversas modalidades de arte rupestre. Ejemplo de ello lo constituirían las diferencias formales entre los motivos geométricos complicados en el norte y los motivos grabados y pinturas abstractos-representativos en el sur; esas diferencias observadas expresarían diversidad étnica entre esas poblaciones (Gradín 1984).

\section{La reacción en contra de la explicación histórico-cultural. Etnohistoria e historia indígena}

Frente al panorama histórico planteado se desarrollaron varias propuestas de superación, una de ellas es la surgida desde los estudios etnohistóricos, concebidos como la historia del momento de contacto entre europeos e indígenas y las consecuencias de este contacto sobre las culturas participantes. Quienes trabajan desde esta perspectiva reclaman la historicidad de los pueblos americanos y rechazan la diferenciación y separación entre la historia de las civilizaciones grafas y la historia de las sociedades ágrafas no estatales (Bechis 1992; Palermo 2000; Nacuzzi 1998). Por otro lado, desde los denominados «estudios de historia indígena», críticos de la denominación Etnohistoria objetan el interés por la fragmentación disciplinar de acuerdo con un problema, en este caso el período de contacto, por volverlo difuso y confuso (Mandrini 1992; Ortelli 1996; Vezub 2005). Consideran que la historia de los pueblos indígenas debe ser abordada desde campos multidisciplinares como el histórico, arqueológico y antropológico, evitando la construcción de una disciplina basada en la otredad. Proponen una mirada más dinámica del mundo indígena, incorporando los análisis sobre etnogénesis y mestizaje, introduciendo categorías de análisis económicas y antropológicas, para superar el paradigma descriptivo dominante en las historias nacionales.

Una lectura antropológica de las fuentes históricas y arqueológicas fue realizada por el arqueólogo A. Rex González ${ }^{6}$, quien a pesar de su breve paso por la arqueología patagónica planteó, en su trabajo «Las exequias de Paine Guor. El suttée entre los araucanos» (1979), que la presencia de determinados rasgos culturales como el sutte 7 , indicarían el resurgimiento de una vieja costumbre presente en los señoríos del siglo XVIII. Ésta estaría expresada a través de la concentración de riqueza, el liderazgo hereditario y los privilegios gozados por los caciques. Este trabajo inspiró en gran medida los trabajos del historiador Raúl Mandrini, pero su propuesta no proliferó en el campo arqueológico (Boschín y Llamazares 1986). Mandrini, desde el estudio de las bases económicas, intenta analizar el proceso de complejización social; considera que los grandes circuitos mercantiles y ganaderos indígenas establecidos durante el siglo XIX unían a las poblaciones a ambos lados de la cordillera abasteciendo a los mercados coloniales. Esta amplia red de intercambio estaría conformada por diferentes núcleos de producción, intercambio y movilización de recursos basados fundamentalmente en

\footnotetext{
${ }^{6}$ Discípulo de Julian Steward, y formado dentro de la Escuela de la Ecología Cultural Norteamericana, ejecutó una labor difícilmente superable en el campo de la arqueología de los Andes argentinos.

${ }^{7}$ Refiere a la matanza de mujeres en los funerales de un cacique araucano como ofrenda al muerto o por motivos de brujería.
} 
una economía pastoril (Mandrini 1988, 1992). Establece que el control de este circuito estaba en manos de cacicatos que legitimaban su poder sobre la base de la pertenencia a una elite familiar, poseyendo atribuciones de liderazgo político, de intermediación con las autoridades criollas y de redistribución de bienes (Mandrini y Paz 2002). Adan Hadyuk y Ana Albornoz (2001) han publicado evidencias arqueológicas en este sentido. Ambos analizaron arqueológica e históricamente los pasos cordilleranos del noroeste patagónico, conocidos como Camino de las Lagunas, que atravesaban áreas terrestres y lacustres. A partir del uso de fuentes históricas - principalmente las producidas por misioneros- intentan diferenciar las etnias que habitaron en el período comprendido entre los siglos XVI y XVII la región del lago Nahuel Huapi. Los autores escriben acerca de la identificación étnica y de la comparación descriptiva que hace a los componentes arqueológicos hallados, identificando la presencia y ausencia de rasgos guías. Es precisamente la identificación de rasgos guía lo que les permite hacer comparaciones con sitios de contacto y precontacto, con el fin de definir un área de intercambio cultural. Arqueológicamente, los trabajos de Ana Biset y Gladys Varela (1991) sobre la excavación de un cementerio indígena del siglo XVIII correpondiente al sitio Caepe Malal (Neuquén), intentan confrontar la evidencia material producto de las excavaciones con la interpretación histórica propuesta por Mandrini, dado que este sitio presentaba abundante material europeo e indígena. La consecuente explicación intentó superar la mera descripción de etnias diferentes y el intercambio de objetos entre ellas, interpretando el material arqueológico «exótico» en función de su intermediación entre las economías capitalistas coloniales de Buenos Aires y Santiago de Chile. El abundante material asociado a los entierros permitió identificar diferencias, proponiendo que el sitio representaría un período de transición hacia los grandes cacicatos del siglo XIX.

La dinámica social de los momentos de contacto fue entendida en parte con relación a la adopción de un nuevo medio de producción, como fue el caballo europeo domesticado. La búsqueda de elementos relacionados con el «complejo ecuestre» que describe la etnografía fue uno de los objetivos centrales: registrar la presencia de caballo como elemento diagnóstico de caza y pastoreo, armas que sustituyen el arco y la flecha, toldos y vestimentas elaborados con cuero de caballo, y expansión de la lengua mapudungun. Esta denominación de «complejo ecuestre» fue rechazada por Miguel Ángel Palermo, quien entendió como una postura simplista y reduccionista el considerar la introducción del caballo como principal factor de cambio social. Es por ello que propone analizar las bases económicas del cambio, reconstruyendo los circuitos de intercambio de sociedades que él define como ganaderas (Palermo 1986). Marca una diferencia substancial entre los tehuelche meridionales y septentrionales, a la vez que señala que los primeros continuarían siendo nómadas cazadores de guanacos a lo largo del siglo XIX que habrían introducido el caballo tardíamente, potenciando su modelo económico. Por el contrario, las transformaciones económicas sucedidas en el segundo grupo resultarían ser muy marcadas, pues la introducción del caballo implicó nuevos aportes a la dieta, influyendo en la producción ganadera, equina y bovina, $\mathrm{y}$ en el intercambio regional de ganado.

Otra de las problemáticas analizados desde el campo de la etnohistoria es la constituida por los procesos de etnogénesis acaecidos en el norte patagónico a mediados 
del siglo XIX; considerando que el avance del Estado y el incremento en el nivel de conflicto habría disparado los mecanismos de fisión, migración y conformación de jefaturas. El concepto de cambio social es entendido como el resultado del incremento de la violencia estatal, la desarticulación de la organización política de las etnias procedentes del período del precontacto y la necesidad de generar y consolidar nuevas estructuras políticas de asociación, resistencia y coalición (Nacuzzi 1993-94, 1998; Vezub 2005).

Desde otra perspectiva, Teresa Boschín (2002) cuestiona los esquemas generales de clasificación étnica aplicados al período temprano postconquista propuestos desde la etnología histórico-cultural para Patagonia norte. Considera que el grado de generalización de estos esquemas no permite analizar las particularidades de los fenómenos socioculturales, por lo que propone analizar diversos «círculos de etnicidad», en ciertos casos superpuestos debido a las consecuencias de la intensificación de los intercambios y de la circulación de bienes e ideas entre grupos diversos. Así por ejemplo, algunos de esos círculos podrían coincidir con la territorialidad de cada una de las lenguas, que estarían en el interior de otro círculo más amplio, y por tanto más laxo y heterogéneo, que se configuró como resultado de la adopción de la lengua gununa iajich como lengua franca en el centro-norte de Patagonia desde el siglo XVIII. Por otro lado, esos círculos de etnicidad más amplios y laxos podrían explicar la coexistencia de diversos estilos de arte rupestre, identificables arqueológicamente. Las semejanzas estilísticas explicarían la adopción de elementos iconográficos, transfiriéndose posteriormente a otros soportes, como los tatuajes, la decoración corporal y la iconografía textil (Boschín 2007).

El principal aporte de estos nuevos enfoques históricos ha sido la profundización histórica de la dinámica de los procesos de diferenciación social y la evaluación de los efectos del proceso de contacto sobre el desarrollo de la diversidad, la desigualdad y la resistencia étnica.

\section{La arqueología procesual y el neoevolucionismo en la arqueología patagónica}

Una de las corrientes teórico metodológicas de mayor preponderancia dentro de la arqueología patagónica corresponde a la designada como Nueva Arqueología o Arqueología Procesual. Surgida a fines de los años de 1970 en Estados Unidos y basada en la epistemología del neopositivismo lógico hempeliano, proclamaba una conversión de la disciplina hacia las ciencias naturales, centrando los estudios arqueológicos en las estrategias adaptativas de las sociedades. Critica la preponderancia dada por la Escuela Histórico-Cultural a la atemporalidad del concepto de etnia y de las formas de poblamiento, a la vez que señala el error que supone no considerar las diferencias funcionales o etapas de procesamiento (económico) de un grupo humano como posible explicación de la variabilidad del registro arqueológico. Entendiendo a la cultura como un sistema adaptativo, explican las relaciones espacio-temporales a través del funcionamiento de los diferentes niveles que componen un sistema social. La explicación del cambio se centra así, en los factores extrínsecos (cambios ambientales) e intrínse- 
cos (maximización de beneficios económicos y minimización de riesgos) tendentes a buscar el equilibrio dentro del sistema cultural. Es precisamente la pérdida de ese equilibrio logrado, debido a causas tecnológicas, ambientales o demográficas, la determinante del cambio social (Aschero et al. 1983; Belardi 2003; Borrero 1994-95, 1995; Mengoñi Goñalons 1988; Moreno 2008; Orquera 1987).

Desde los años ochenta, el paradigma procesual en la arqueología patagónica ha sido preponderante. Sus aportes enriquecieron la disciplina primordialmente en el campo metodológico, convirtiendo a las especializaciones como la arqueozoología, paleobotánica, geoarqueología y tafonomía en las líneas cardinales de las investigaciones. Estas vías de trabajo acrecentaron una producción arqueológica que, a causa de la fuerte hegemonía que tuvo durante cuarenta años la escuela histórico-cultural, permitió superar lo que algunos investigadores denominaron «el factor retardatario» de la arqueología patagónica (Boschín y Llamazares 1986).

Desde la arqueología procesual se asume que los cambios en la organización social y económica estarían motivados por causas extrínsecas a lo social. Ejemplo paradigmático de esta postura es el estudio del posible cambio económico producido durante la Anomalía Climática Medieval ocurrida entre el 1021-1228 d.C. (Stine 1994, 2000), provocando situaciones de estrés ambiental cuyas consecuencias sociales conducirían a definir nuevas estrategias como migración, conflicto y aumento del desarrollo tecnológico, mediante el cual se pudieran maximizar los recursos disponibles (Rafferty 1985). Rafael Goñi y Gustavo Barrientos (2004), en su análisis sobre el poblamiento del Holoceno Tardío en la cuenca del lago Salitroso en la Provincia de Santa Cruz, consideran que las características ecológicas de diferentes ambientes condicionarían la distribución de los grupos humanos, su movilidad y el acceso a los recursos. Durante el cambio climático del 1021-1228 d.C. los recursos críticos se habrían restringido llevando a las poblaciones a permanecer concentradas en el espacio. Bajo esta óptica, las decisiones humanas aparecerían determinadas por el clima. Datos arqueológicos como la concentración de tumbas en un área específica reflejarían de qué modo las acciones humanas hubieron respondido a las determinaciones climáticas y ecológicas. Del mismo modo, se interpreta que los cambios climáticos provocaron mutaciones en las adaptaciones, así como cambios en la distribución de los recursos alimenticios actuando como factor de extinción de las poblaciones humanas.

Un problema que surge ante esta perspectiva es que en ocasiones se cuestiona la continuidad histórica de los pueblos patagónicos, rechazando las interpretaciones que vinculan como descendientes directos a los pobladores actuales e históricos con los del Pleistoceno Final. Menos maximalista, Borrero (2001) ha supuesto formas de «contacto indirecto» entre las poblaciones indígenas como el principal mecanismo de cambio de las poblaciones históricas. Éstas habrían recibido influencias de otras etnias que mantendrían contactos con la colonia, lo que sumado a enfermedades infecciosas que pudieron diezmar poblaciones, uniones entre diferentes etnias y una presunta transformación biológica de las poblaciones originales, habría causado la desintegración de la sociedad indígena y la falta de continuidad directa entre las sociedades arqueológicas e históricas.

La arqueología evolutiva ha intentado superar el enfoque procesual explicando el cambio a partir de las variaciones registradas al nivel del genotipo y del fenotipo, los 
mecanismos evolutivos de dispersión, variación, selección, vicariancia y competición permitirían explicar los procesos de evolución multidireccional, desvinculándolos de la tradicional idea antropológica que prioriza el factor humano en la explicación de las transformaciones sociales (Lanata 1996, 2002). Las entidades humanas son entendidas como entes biológicos, por lo tanto la extinción poblacional es una forma de explicar la desaparición de los grupos humanos. Así se ha inferido la expansión de poblaciones desde el nordeste patagónico hacia la región pampeana y cuyana (Barrientos y Pérez 2004) y el mestizaje durante el Holoceno Tardío Final entre grupos de la precordillera norpatagónica, del nordeste de Río Negro y de la Región Pampeana (González-José et al. 2004). Del mismo modo se han llevado a cabo análisis referentes a ciertas lesiones óseas identificadas en cráneos del norte patagónico. El resultado de dichos análisis ha llegado a sugerir un posible aumento en las lesiones craneales para momentos tempranos de contacto y producidas por armamento de origen europeo (Barrientos y Gordón 2004). Nuevos estudios mostrarían que ese aumento en la violencia coincide con el momento del contacto, dado el predominio de heridas producidas por instrumentos metálicos (Gordón y Ghidini 2007).

El estudio arqueológico de las formas de interacción entre grupos humanos diferenciados geográficamente y las redes de intercambio indígenas en el periodo inmediatamente anterior a la conquista constituye uno de los temas de investigación que más interés ha suscitado en los últimos años. Los trabajos arqueológicos de Goñi (1986-87) en el río Malleo, Neuquén, intentan explicar el rol del caballo dentro del circuito comercial con Chile y cómo este nuevo escenario permitió extender la territorialidad de las bandas de cazadores recolectores, incorporando zonas con acceso permanente a agua y pastos, y modificando los tiempos de ocupación del territorio. Julieta Gómez Otero (2007), por su parte, sugiere la modificación de las formas de relación entre grupos al final del periodo, cuando las redes de intercambio se habrían extendido, como lo demuestra el hacha de bronce encontrada en la desembocadura del río Chubut a $2.000 \mathrm{Km}$. del lugar de su producción, en el noroeste argentino (Gómez Otero y Dahinten 1997-98) o el hallazgo de molinos elaborados con granitos cuyas fuentes se encontrarían a más de $100 \mathrm{Km}$ de la costa. Enfatiza la relación costebeneficio entendiendo que al incrementarse la demanda aumentaría la recolección, el tiempo de trabajo y la especialización, generando de este modo un excedente y un consecuente aumento en la complejidad social. Desde una postura procesual arriesga una hipótesis sobre el Periodo Tardío, concibiendo que la diferenciación social adquiriría matices funcionales; en otras palabras, el cambio ambiental generaría estrés-conflicto y de éste emergería como resultado el liderazgo.

Cabría preguntarse si estos nuevos planteamientos han significado un cambio en cuanto a la concepción esencialista de la cultura. El hecho de suponer la extinción de una población por causa de enfermedad, o bien uniones étnicas o transformaciones biológicas, implica que la vinculación entre la cultura y el sustrato biológico se sigue manteniendo. Las causas sociales de las transformaciones observadas siguen sin explicarse. Afirmar que la diferenciación social puede ser identificada a partir de elementos diagnósticos en el registro no implica una profunda diferenciación del paradigma histórico cultural. Paradójicamente, la vinculación entre arqueología y antropología biológica se ha reforzado en este período, pero las relaciones entre las dis- 
ciplinas sociales de antropología, historia o etnografía se enfriaron, no por la falta de planteamientos interdisciplinares, sino más bien por la falta de incorporación de teoría social en la práctica arqueológica. La adopción de teorías, conceptos y categorías de las ciencias naturales eclipsaron o, en sus propios términos, mutaron el objeto de estudio de la arqueología sin superar los argumentos histórico-culturales, pues se continúan buscando (o encontrando) las causas del cambio en las causas externas a los fenómenos sociales. El plantear una reconstrucción de una historia ecológica no significa sólo una deshumanización del proceso histórico; implica además un distanciamiento del papel de historiadores y una desvinculación de la arqueología con la sociedad actual. Negar la vinculación histórica de las sociedades indígenas actuales con las prehistóricas significa reproducir, legitimar y naturalizar al indígena en su subalternidad.

\section{8. ¿Es posible una interpretación alternativa en la arqueología de Patagonia?}

Muchas de las propuestas que se han mencionado no sólo intentaron superar el paradigma anticuarista e histórico-cultural, sino que también intentaron evitar el reduccionismo de las interpretaciones simplistas de los fenómenos sociales, que sólo tienen en consideración los cambios ambientales, el crecimiento demográfico y determinismos geográficos y/o económicos.

Cabe señalar que cuando los historiadores o etnohistoriadores cuestionaron que una realidad histórica no podría ser analizada si no se integraba el mundo indígena a ese contexto, la arqueología debió haberse posicionado como el principal aporte a este problema, pero la arqueología patagónica no asumió tal papel. Si la reconstrucción de la historia indígena patagónica sigue siendo un fenómeno ajeno a la historia nacional, entonces los arqueólogos, como agentes del Estado, ¿seguimos negando la historia de aquellos a los que seguimos considerando «otros», afirmando implícitamente la idea de una región que «se vació» y se convirtió en «desierto» por sí misma? Plantear esta pregunta significa cuestionarnos en qué se diferencia nuestra propia producción y práctica arqueológica del tan cuestionado anticuarismo de principios del siglo XX, en cuanto a la relación y el compromiso que establecemos como investigadores con la historia social de una región. La búsqueda de criterios arqueológicos objetivos para conceptualizar trayectorias históricas ha limitado la comprensión de los procesos a aquellos aspectos relacionados con la diferenciación estilística, la distribución y trazabilidad de esos rasgos. Los criterios empleados se fundamentan principalmente en explicaciones geográficas, cronológicas y morfológicas, sin abordar las nociones de grupo social y de actividades sociales como agentes factibles de transformación de los registros encontrados y no como meros receptores de las condiciones de las dinámicas sociales.

¿Hay una alternativa? Creemos que sí, y pasa por describir a las sociedades patagónicas en términos de la históricamente cambiante trama de actividades sociales (de subsistencia, producción y reproducción) y de las relaciones y vinculaciones que éstas generaron. Entre aquellos factores fundamentales que debiéramos tener en cuenta y que sistemáticamente son olvidados, podríamos enumerar las estimaciones demográ- 
ficas (mortalidad, población, densidad de poblamiento en áreas diversas), que pueden llevarse a cabo a partir de datos arqueológicos, pero sin olvidar la necesaria integración con informaciones de naturaleza más biológica, como sería la estimación del número de hombres y mujeres en edad productiva y reproductiva, del número mínimo de unidades mínimas de reproducción («familias»), de la composición de unidades mínimas residenciales, entre otras estimaciones posibles.

Mucho se ha avanzado en la documentación del medio físico y su historia geomorfológica y ambiental, pero sería necesario integrar esa información con una discusión acerca de las necesidades de subsistencia de la población y los recursos disponibles explotables con diferentes grados de inversión de trabajo. Al mismo tiempo, no debiéramos olvidar que las poblaciones humanas no son meros estómagos bípedos, sino que existen y son objetivamente analizables las necesidades de mantenimiento, de vinculación social, de movilidad, en una palabra, de reproducción social. Por todo ello una historia social de la Patagonia, desde sus inicios hasta el presente, no debiera limitarse a buscar posibles discontinuidades en el poblamiento o aparentes cambios económicos correlacionados con fenómenos climáticos o con la llegada de nuevas poblaciones. «Evolución» no significa necesariamente una linealidad de diversos estadios ordenados desde el más simple (y antiguo) al más complejo (y moderno). Por el contrario, debemos documentar la extrema variabilidad de soluciones locales adoptadas por diversas comunidades humanas (no diversas «etnias») y estudiar cómo los cambios en la forma de gestión de la subsistencia estuvieron o no relacionados con cambios en la gestión de la movilidad económica o social, con transformaciones en los mecanismos de gestión de la reproducción social, etc.

Más allá de materialismos economicistas triviales y simplistas, una historia alternativa de la Patagonia debiera estar encaminada al estudio de cómo los grupos humanos que allí vivieron y se reprodujeron fueron capaces de producir información acerca de su entorno y acerca de los otros grupos humanos con los que se relacionaron. Ningún grupo ha podido subsistir aisladamente; las distintas formas de interacción social, ya sea exogamia, comercio, guerra, etc. constituyen los mecanismos básicos de transformación social, y ninguno de ellos se puede producir sin que los individuos implicados no hubiesen producido conocimiento social (información).

Es posible que la investigación arqueológica no pueda proporcionarnos toda la información primaria que necesitaríamos para realizar una fotografía precisa de las formas de vida en el pasado de la Patagonia. Sin embargo, sí que puede proporcionarnos una explicación objetiva de las formas de movilidad de los grupos humanos en el espacio, así como de los condicionantes sociales (y no sólo físicos) que hubiesen influido en esa movilidad. Entre ellos debemos mencionar las diferentes formas de poder (coerción, coacción, convencimiento) y sus consecuencias en la conformación de jerarquías sociales y políticas. La perspectiva neoevolucionista, junto con la incorporación de la teoría de sistemas, permitió comprender la variabilidad espacio-temporal de los procesos sociales y trascender los esquemas tipológicos tradicionales que equiparaban a la cultura con los objetos arqueológicos; sin embargo la falta de una teoría social que enfatizara los aspectos más dinámicos de una sociedad y, en especial, las diferentes formas en que una sociedad interactúa con otras han obstaculizado el ulterior desarrollo de esta perspectiva. 
El estudio cuantitativo de todos estos rasgos, la búsqueda en unos casos de regularidades probabilísticas, en otros de discontinuidades significativas, nos permitiría reconstruir el proceso de institucionalización social, llegando a poder explicar cómo una población original decidió por sí misma cambiar la manera en que tomaba decisiones sociales que afectaban a su subsistencia, a su reproducción social. A lo largo del tiempo cambió la manera de trabajar, de producir, de acumular, de intercambiarse cosas y gentes, de relacionarse, pero esos cambios no fueron ciegas adaptaciones a un paisaje omnímodo. Fueron decisiones racionales de poblaciones a las que hoy llamamos indígenas y a quienes todavía hoy se les niega la capacidad de entender sus propios problemas y de encontrar nuevos mecanismos de cambio y transformación.

\section{Conclusiones}

Consideramos innegable el hecho que tanto desde el paradigma histórico cultural como desde el neoevolucionista se explicó e interpretó a las sociedades patagónicas como conjuntos cerrados, ya sea cultural, económica y/o étnicamente. Precisamente esas tipologías étnicas continúan vigentes como consecuencia de modelos explicativos de índole descriptivista, cuyos observables, ya sean conjuntos antropológicos o arqueológicos (generalmente conjuntos materiales) se explican por referencia a una tipología preexistente cuya lógica interna nunca ha sido suficientemente puesta de manifiesto.

Lamentablemente, aún cuando la crítica a los rótulos étnicos parece ser casi unánime hoy en día, sigue subyacente en una mayoría de estudios el esquema etnográfico clásico basado en la noción de cultura «esencial» y de rasgos culturales característicos y diferenciales como uno de los elementos fundamentales para la definición étnica (del Castillo 2008).

A nuestro entender, la principal dificultad reside en la propia definición de cultura como sistema adaptativo, que sólo entiende como social los fenómenos derivados de las explicaciones sobre la subsistencia y del subsistema tecnológico. El concepto de cazador-recolector perpetúa la idea de tipo, equiparándolo al nivel de cultura (o incluso a nivel de especie animal). Actualmente, aunque quizás no intencionadamente, se continúa asumiendo que el concepto cazador recolector reúne o sintetiza rasgos concebidos como universales y homogeneizados. Si la interpretación histórico-cultural cae en un reduccionismo tipológico que constriñe a las culturas en variedades artefactuales, la explicación ecologicista acaba en otra forma de reduccionismo que limita a las culturas a sistemas conductuales adaptativos universales.

Trasladar los modelos evolutivos simplistas de la antropología tradicional, realizar acríticamente analogías interculturales para contrastar modelos de comportamiento (etnoarqueología) y delimitar una unidad de análisis denominada cazadora-recolectora como estadio evolutivo (y no entendida como una categoría económica), conlleva al desarrollo de una disciplina tan ahistórica como la que se intentaba criticar desde el neoevolucionismo. Debemos tener en cuenta que las dinámicas de cualquier proceso histórico no son el resultado de la mera superposición de hechos y momentos medianamente definidos, sino que se sustentan en la objetivación de la realidad histórica y 
en la búsqueda causal de la acción transformadora de las sociedades humanas (Barceló, Briz y Vila 1999).

Actualmente las investigaciones arqueológicas acentúan los aportes de nuevas metodologías y técnicas que potencien la información que pueda obtenerse acerca de la empiria arqueológica, pero, más allá del enorme avance metodológico en numerosas subdisciplinas y especialidades con gran potencial surgidas recientemente, no existe un correlato respecto de ampliar las posibilidades de interpretación social del registro. Ello deriva lamentablemente en una confusión del objeto de la práctica arqueológica como el objetivo de la explicación histórica. Es precisamente esta confusión de no saber si perseguimos como último fin el objeto o el objetivo, lo que ha llevado a que numerosos proyectos arqueológicos se desarrollen al margen de una auténtica problemática histórica y sin problemas sociales concretos más allá de obviedades no siempre certeras que resolver al momento de iniciar las investigaciones. De algún modo, ha llegado a reconocerse erróneamente que como ya conocemos lo conocible acerca de cazadores-recolectores, el campo de conocimiento que queda por profundizar se ciñe a los mismos objetos arqueológicos y a cómo estos han llegado a aparecer donde aparecen dentro del contexto de descubrimiento. Esta actitud como fin último de trabajos de investigación resulta en reducir la meta arqueológica al planteamiento de problemáticas vinculadas a la naturaleza del registro y a procesos de formación y transformación del registro arqueológico.

En contra del evolucionismo social clásico no debiéramos imaginar a las sociedades cazadoras-recolectoras en la base de una pirámide o en el punto de partida de una escala de desarrollo posterior. Se trata de sociedades diferentes de aquellas que construyen su economía sobre la base del control y la manipulación de la reproducción vegetal y animal. Esa diferencia no debe leerse en términos de tipos excluyentes, sino que debe ser entendida como una gradación, un continuo. Para definir y caracterizar una sociedad cazadora-recolectora resulta fundamental considerar los factores y parámetros que permitan cuantificar la intensidad y la finalidad con que los agentes sociales que la conforman realizan diferentes tipos de acciones.

Si la sociedad industrial contemporánea no puede ser descrita únicamente en términos de lo que come y construye, tampoco podemos simplificar de ese modo las sociedades no industriales. Lo que comemos y cómo comemos está siempre mediado por el contexto social en el que producimos aquello que comemos y en el que interactuamos con otras personas, así como por la manera cómo nos reproducimos, obviamente en tanto que seres vivos, pero también como miembros de una comunidad que tiene una manera concreta de hacer lo que hace. Más que describir un grupo humano por lo que come debemos caracterizarlo por cómo trabaja y por cómo se relaciona con otros grupos geográficamente más o menos próximos, ya sea colaborando en reciprocidad, ya sea imponiéndose o condicionando a otro grupo.

Las descripciones arqueológicas de los grupos humanos patagónicos han sido reducidas a la relación hombre-medioambiente, entendidas éstas desde una perspectiva funcional que concibe la economía en términos de la optimización de las adaptaciones de comunidades, las cuales se adaptan a ecosistemas particulares y se extinguen si no son capaces de hacerlo. En escasas ocasiones se contempla cómo el trabajo invertido 
en actividades no directamente relacionadas con la subsistencia influyen, condicionan o determinan la misma apropiación de recursos y su transformación en productos.

Es cierto que la metodología arqueológica limita y condiciona el estudio de la trayectoria histórica de las sociedades patagónicas. No obstante no siempre se ha sabido interpretar la evidencia arqueológica disponible porque no se formulan las preguntas que se debieran formular. Es cierto que no podemos averiguar cómo pensaban, pero sí resulta factible desarrollar técnicas de análisis que nos permitan saber cómo producían (y no sólo qué), cómo consumían (y no sólo qué) y cómo se organizaba socialmente la producción con vistas a su consumo.

Por descontado, el posible avance de la investigación no pasa por el rechazo sin más de la inmensa base de conocimientos que se ha generado hasta hoy. Debiéramos buscar medios que, aunque mecánicos, sean prácticos y eficientes y nos permitan integrar distintos procesos causales. Se trata de integrar distintas explicaciones que pueden ser parcialmente correctas pero que por sí solas resultan insuficientes, cuando no incompletas o distorsionadas, respecto de la realidad pasada que pretendemos conocer. Es por ello que nuestra propuesta de abordaje de los mecanismos que generan la diversidad y la homogeneización social patagónica contempla la aplicación de técnicas de simulación computacional basada en agentes que reproducen la interacción entre comunidades cazadoras-recolectoras (Barceló, del Castillo, Mameli y Moreno 2009).

El principal interés que nos ha motivado a aplicar esta metodología experimental es la exploración de las implicaciones arqueológicas de los modelos arqueológicos y etnográficos conocidos, con el fin de identificar los rasgos y variables que caracterizan la complejidad de los sistemas culturales. No pretendemos representar artificialmente a una sociedad cazadora recolectora mediante la suma descriptiva de la empiria disponible, ni realizar una representación del funcionamiento social. Nuestro objetivo es principalmente heurístico, lo cual nos permitirá aproximarnos a la elaboración de nuevos principios explicativos de la etnicidad en arqueología. La integración de diferentes posturas teóricas, con el propósito de experimentar con ellas, nos permitirá comprender cómo desde los factores propuestos (sean éstos ambientales, sociales y/o materiales) las respuestas de los agentes sociales han sido condicionadas y cómo estas respuestas modelan los comportamientos colectivos. El fundamento de nuestro enfoque es que decisiones en las que derivan cambios visibles arqueológicamente fueron socialmente mediadas y no ecológica o ambientalmente impuestas: por lo tanto, debemos desarrollar metodologías que nos permitan percibir en el registro arqueológico el cambio social y la complejidad de las relaciones entre personas.

Más allá de la complejidad del desarrollo matemático, epistemológico y tecnológico que conlleva el trabajar con simulación computacional, la evolución de estas técnicas las ha vuelto más accesibles a la investigación, posibilitando su aplicación en el ámbito de las ciencias sociales y más recientemente en el campo arqueológico. La modelización basada en agentes es una metodología que permite el estudio de dinámicas culturales no lineales que emergen de las acciones históricas de agentes heterogéneos que interactúan en un espacio definido. Cada agente es un programa de software autónomo que actúa, interactúa y responde a su entorno virtual, conformado por otros agentes. Producto de este proceso de interacciones entre agentes y acciones de agentes emerge un comportamiento global del sistema, es decir, emergen patrones, estructuras 
o propiedades que sólo pueden explicarse en términos de componentes e interacciones y que no pueden ser reducidas a las acciones individuales. Así, las sociedades artificiales proveen nuevas perspectivas sobre la emergencia social cuya explicación debe ser entendida como la dialéctica entre la emergencia social y la causación social.

Dado que en nuestro caso nos interesa simular la trayectoria histórica del poblamiento en Patagonia, estos resultados son altamente relevantes para nuestros propósitos, proponiéndonos comenzar justo allí donde han acabado otras investigaciones. La interacción entre el entorno físico y los agentes sociales es ahora bien entendida, pero necesitamos mucho más trabajo teórico y metodológico para implementar formas sociales de interacción, así como la emergencia de contradicciones sociales a lo largo de una trayectoria histórica que hasta hace muy poco se consideraba estable y homogénea a lo largo de más de 10.000 años. La propuesta de introducir nuevos métodos analíticos como es el caso de la simulación en arqueología no sólo implica un nuevo aporte metodológico, también implica la posibilidad de reinterpretar los clásicos esquemas etnológicos y las clasificaciones arqueológicas bajo la posibilidad de la experimentación teórica.

\section{Referencias bibliográficas}

AmBrosetTi, Juan. B.

1903 «Las grandes hachas ceremoniales de la Patagonia». Anales del Museo Argentino de Ciencias Naturales 3: 45-51.

Aschero, Carlos, Cecilia Pérez de Micou, Lidia Nacuzzi, María Onetto, Cristina BelleLLI, Alfredo Fisher y Raúl SCANDROGLIO

1983 Arqueología del Chubut. El Valle de Piedra Parada. Rawson: Dirección Provincial de Cultura del Chubut.

BARCELó, Joan A., Ivan BRIZ y Assumpció ViLA

1999 «New Techniques for Old Times. A General Introduction to Computing Archaeology», en New Techniques for Old Times. Computer Applications and Quantitative Methods, pp. 3-17. BAR International Series 757. Oxford: Archaeopress.

Barceló, Joan A., Ma Florencia del Castillo, Laura Mameli y Julián E. Moreno

2009 «The Computer Simulation of Social Dynamics and Historical Evolution. The Case of «Prehistoric» Patagonia», en Proceeding of the $2^{\text {nd }}$ Workshop on Social Simulation and Artificial Societies Analysis (SSASA'08), F. J. Miguel Quesada, ed. CEUR Workshop Proceedings. Documento electrónico, <http://CEUR-WS.org/Vol442/p3BarceloDelCastilloMameliMoreno.pdf>, con acceso el 2/12/2010.

Barceló, Joan A, Ma Florencia del CAstillo, Laura Mameli, Julián E. Moreno y Blanca VIDELA

2009 «Where does the South Begin? Social Variability at the Southern top of the World». Artic Anthropology 46 (1-2): 50-71.

BARRIENTOS, Gustavo y Florencia GoRDÓN

2004 «Explorando la relación entre nucleamiento poblacional y violencia interpersonal durante el Holoceno tardío en el noreste de Patagonia (República Argentina)». Magallania 32: 53-69. 
BARrientos, Gustavo e Iván PÉREZ

2004 «La expansión y dispersión de poblaciones del norte de Patagonia durante el Holoceno tardío: evidencia arqueológica y modelo explicativo», en Contra viento y marea. Arqueología de Patagonia, M. T. Civalero, P. M. Fernández y A. G. Guraieb, eds., pp. 179-195. Buenos Aires: INAPL-SAA.

BARTOLOMÉ, Miguel A.

2004 «Los pobladores del «desierto». Genocidio, etnocidio y etnogénesis en la Argentina». Amérique Latine Histoire et Mémoire. Les Cahiers ALHIM 10. Documento electrónico, <http://alhim.revues.org/index103.html>, con acceso el 2/12/2010.

BECHIS, Martha

1992 «Instrumentos para el estudio de las relaciones interétnicas en el período formativo y de consolidación de los Estados nacionales», en Etnicidad e Identidad, C. Hidalgo y L. Tamagno, pp. 82-108. Buenos Aires: CEAL.

BELARDI, Juan B.

2003 Paisajes arqueológicos: un estudio comparativo de diferentes ambientes patagónicos. Tesis Doctoral, Universidad de Buenos Aires.

Biset, Ana M. y Gladys VArela

1991 «El sitio arqueológico de Caepe Malal. Una contribución para el conocimiento de las sociedades indígenas del noroeste neuquino en el siglo XVIII», en Cuadernos de Investigación. Arqueología y Etnohistoria de la Patagonia Septentrional, M. T. Boschín, comp., pp. 36-48. Tandil: IEHS.

BÓRMIDA, Marcelo

1953-54 «Los antiguos patagones. Estudio craneológico». Runa 6: 1-95.

1976 Etnología y Fenomenología: Ideas acerca de una hermenéutica del extrañamiento. Buenos Aires: Cervantes.

Borrero, Luis A.

1994-95 «Arqueología de Patagonia». Palimpsesto 4: 9-56.

1995 «Historia reciente de la arqueología patagónica». Runa 22: 151-176.

2001 El poblamiento de la Patagonia. Toldos, milodones y volcanes. Argentina: Emecé.

Boschín, $\mathrm{M}^{\mathrm{a}}$ Teresa

2002 «Indigenous History of Northwest Patagonia: Regional Identities during the Seventeenth and Eighteenth Centuries», en Archaeological and Anthropological Perspectives on the Native Peoples of Pampa, Patagonia, and Tierra del Fuego to the Nineteenth Century, C. Briones y J. L. Lanata, eds., pp. 75-88. Westport: Bergin and Garvey.

2007 Identidad, territorialidad e ideología de las sociedades de cazadores-recolectores (3.000 AP-1.400 AP) de la Patagonia argentina. Arte rupestre del ámbito estepario septentrional en las subcuencas de los arroyos Pichileufú, Comallo y Maquinchao. Tesis Doctoral, Universidad de Salamanca.

Boschín, M ${ }^{\mathrm{a}}$ Teresa y Ana $\mathrm{M}^{\mathrm{a}}$ LLAmazARes

1986 «La Escuela Histórico-cultural como factor retardatario del desarrollo científico de la arqueología argentina». Etnia 32: 101-156.

BRUCH, Carlos

1902 «La piedra pintada del arroyo Vaca Mala y las esculturas de la cueva de Junín de los Andes (Territorio del Neuquén) ». Revista del Museo de La Plata 10: 173-176. 
CASAmiquela, Rodolfo M.

1965 Rectificaciones y ratificaciones. Hacia una interpretación definitiva del panorama etnológico de la Patagonia y área septentrional adyacente. Cuadernos del Sur. Bahía Blanca: Universidad Nacional del Sur

1969 Un nuevo panorama etnológico del área pan-pampeana y patagónica adyacente. Pruebas etnohistóricas de la filiación tehuelche septentrional de los querandies. Santiago de Chile: Museo Nacional de Historia Natural.

1985 Bosquejo de una etnología de la provincia de Río Negro. Viedma: Fundación Ameghino.

Cocilovo, José A.

1992 «Biología y cultura: una controversia. 1. Objeta J. A. Cocilovo». Ciencia Hoy 4 (20): 16-19.

Curtoni, Rafael y Gustavo Politis

2006 «Race and Racism in the Archaeology of South America». World Archaeology 38: 93-108.

DE APARICIO, Francisco

1935 «Viaje preliminar de exploración en el territorio de Santa Cruz». Publicaciones del Museo Antropológico y Etnográfico de la Facultad de Filosofía y Letras, Serie A 3: 71-92.

Del Castillo Bernal, Ma Florencia

2008 Revisión crítica de los enfoques arqueológicos, históricos y etnográficos sobre la sociedad indígena patagónica durante el Periodo Postconquista (XVI-XVIII). Tesis de Maestría, Universidad Autónoma de Barcelona.

ESCALADA, Federico

1949 El complejo Tehuelche. Estudios de Etnografía Patagónica. Buenos Aires: Coni.

Gómez Otero, Julieta

2007 Dieta, uso del espacio y evolución en poblaciones cazadoras-recolectoras de la costa centro-septentrional de Patagonia durante el Holoceno Medio y Tardío. Tesis Doctoral, Universidad Nacional de Buenos Aires.

Gómez OTERo, Julieta y Silvia DAHINTEN

1997-98 «Costumbres funerarias y esqueletos humanos: variabilidad y poblamiento en la costa Nordeste de la provincia del Chubut (Patagonia Argentina)». Relaciones de la Sociedad Argentina de Antropología 22/23: 101-124.

Gómez Romero, Juan F.

2007 Sistemas de relaciones sociales en la frontera sur de Buenos Aires: yacimientos Fortín Miñana (1860-1863) y Fortín Otamendi (1858-1869). Tesis Doctoral, Universidad Autónoma de Barcelona.

GonZÁLEZ, Alberto R.

1979 «Las exequias de Paine Guor. El suttée entre los araucanos de la llanura». Relaciones de la Sociedad Argentina de Antropología 13: 137-161.

GonzÁlez-José, Rolando, Neus Martínez-Abadías, Silvina van Der Molen,

Clara García-Moro, Silvia DAHINTEN y Miquel HernándeZ

2004 «Hipótesis acerca del poblamiento de Tierra del Fuego-Patagonia a partir del análisis genético poblacional de la variación craneofacial». Magallania 32: 79-78. 
GoÑI, Rafael

1986-87 «Arqueología de sitios tardíos en el Valle del Río Malleo, Provincia del Neuquén». Relaciones de la Sociedad Argentina de Antropología 17 (1): 37-66.

GoÑI, Rafael y Gustavo BARRIENTOS

2004 «Poblamiento tardío y movilidad en la cuenca del lago Salitroso», en Contra Viento y Marea. Arqueología de Patagonia, editado por T. Civalero, P. Fernández y G. Guraieb, pp. 313-324. Buenos Aires: INAPL-SAA.

Gordón, Florencia y Gabriela GHIDINI

2007 «Análisis bioarqueológico de la violencia interpersonal. El valle inferior del Río Negro (República Argentina) durante el Holoceno tardío». Werken 9: 27-45.

Gradín, Carlos

1984 «Investigaciones arqueológicas en «Casa de Piedra», provincia de La Pampa», en Investigaciones Arqueológicas en Casa de Piedra, C. Gradín y otros, eds., pp. 762. La Pampa: Dirección General de Cultura y Ente Ejecutivo Casa de Piedra.

1985 «Área de los cazadores meridionales (Pampa-Patagonia)», en Cazadores de la Patagonia y Agricultores Andinos, J. Schobinger y C. Gradín. Madrid: Encuentro.

HADYUK, Adan y Ana Ma AlBORNOZ

2001 «Antecedentes arqueológicos e históricos del «Camino de las Lagunas»»». Tiempos Patagónicos 3 (7).

HARRINGTON, Tomás

1946 «Contribución al estudio del indio Gününa Küne». Revista del Museo de La Plata 2: $237-275$.

IMBELLONI, José

1949 «Los Patagones. Características corporales y psicológicas de una población que agoniza». Runa 2 (2-3): 5-58.

LANATA, José L.

1996 «Cambios para evolucionar: Las propiedades del registro arqueológico y la evolución de los grupos humanos en Patagonia y Tierra del Fuego», en Arqueología. Sólo Patagonia, J. Gómez Otero, ed., pp. 99-196. Puerto Madryn: CENPAT-CONICET.

2002 «The World's Southernmost Foragers: The Native Diversity of Tierra del Fuego», en Archaeological and Anthropological Perspectives on the Native Peoples of Pampa, Patagonia, and Tierra del Fuego to the Nineteenth Century, C. Briones y J. L. Lanata, eds., pp. 57-73. Westport: Bergin and Garvey.

MANDRINI, Raúl J.

1988 «Desarrollo de una sociedad indígena pastoril en el área intereserrana bonaerense». Anuario IEHS 2: 71-98.

1992 «Indios y fronteras en el área pampeana (siglos XVI-XIX): balances y perspectivas». Anuario IEHS 7: 59-73.

MANDRINI, Raúl J. y Carlos D. PAZ (eds.)

2002 Las fronteras hispanocriollas del mundo indigena latinoamericano en los siglos XVIII-XIX. Un estudio comparativo. Tandil: IEHS- CEHIR-UNCOMAH-UNSur.

Menghin, Oswald F.A.

1952a «Las pinturas rupestres de la Patagonia». Runa 5 (1-2): 5- 22.

1952 b «Fundamentos cronológicos de la prehistoria de Patagonia». Runa 5 (1-2): 23-43. 
1957 «Estilos de arte rupestre en Patagonia». Acta Praehistórica 1: 57-87.

MENGOÑI GoÑALONS, Guillermo L.

1988 «Análisis de materiales faunísticos de sitios arqueológicos». Xama 1: 71-120.

MORENO, Julián E.

2008 Arqueología y etnohistoria de la Costa Patagónica Central en el Holoceno Tardío. Rawson: Secretaría de Cultura del Chubut.

NACUZZI, Lidia

1993-94 «Los cacicatos duales en Pampa-Patagonia durante el siglo XVIII». Relaciones de la Sociedad Argentina de Antropología 19: 135-144.

1998 Identidades Impuestas. Tehuelches, aucas y pampas en el norte de la Patagonia. Buenos Aires: Sociedad Argentina de Antropología.

2005 «El queso y los gusanos en el extremo sur de América. Grupos étnicos, disputas académicas y un juicio por registro de marca». Revista de Indias 65 (234): 427452 .

ORQueRA, Luis A.

1987 «Advances in the Archaeology of the Pampa and Patagonia». Journal of the World Prehistory 1: 333-413.

ORTELLi, Sara

1996 «La ‘araucanización’ de las Pampas: ¿Realidad histórica o construcción de los etnólogos?». Anuario IEHS 11: 203-225.

Outes, Félix

1905 «La edad de la piedra en Patagonia». Anales del Museo Nacional de Buenos Aires 12 (Ser. 3a., T.5): 203-575.

PAlermo, Miguel A.

1986 «Reflexiones sobre el llamado ‘complejo ecuestre' en la Argentina». Runa 16: 157178.

2000 «A través de la frontera. Economía y sociedad indígenas desde el tiempo colonial hasta el siglo XIX», en Nueva Historia Argentina, vol. 1, M. N. Tarragó, ed., pp. 343-382. Buenos Aires: Sudamericana.

RAFFERTY, Janet

1985 «The Archaeological Record on Sedentariness: Recognition, Development and Implications», en Advances in Archaeological Theory and Methods, M. Schiffer ed., pp.113-148. Nueva York: Academic Press.

STINE, Scott

1994 «Extreme and Persistent Drought in California and Patagonia during Mediaeval Time». Nature 369: 546-549.

2000 «On the Medieval Climatic Anomaly». Current Anthropology 41: 627-628.

VezuB, Julio E.

2005 Etnicidady poder en el país de las manzanas. Tesis Doctoral, Universidad Nacional del Centro de la Provincia de Buenos Aires.

Viegas Barros, José P.

2005 Voces en el viento. Raíces lingüísticas de la Patagonia. Buenos Aires: Mondragón. 
VignATi, Milcíades A.

1936 «Las culturas indígenas de Patagonia», en Historia de la Nación Argentina, vol. 1, pp. 591-645. Buenos Aires: Junta de Historia y Numismática Americana.

1944 Antigüedades en la región de los lagos Nahuel Huapi y Traful. La Plata: Universidad de La Plata.

WoLF, Eric

1982 Europe and the People without History. Berkeley: University of California Press. 